Martínez-de-Quel-Pérez, Ó.; Sánchez-Moreno, A.; Zamorano-Feijóo, C.; Ayán-Pérez, C. (2019). Epidemiología lesional en actividades escolares y extraescolares en la Comunidad de Madrid (España) / Sport Injuries in School and Extracurricular Activities in the Community of Madrid (Spain). Revista Internacional de Medicina y Ciencias de la Actividad Física y el Deporte vol. 19 (73) pp. 151-166 Http://cdeporte.rediris.es/revista/revista73/artprevalencia987.htm

DOI: http://doi.org/10.15366/rimcafd2019.73.011

\title{
ORIGINAL
}

\section{EPIDEMIOLOGÍA LESIONAL EN ACTIVIDADES ESCOLARES Y EXTRAESCOLARES EN LA COMUNIDAD DE MADRID (ESPAÑA)}

\section{SPORT INJURIES IN SCHOOL AND EXTRACURRICULAR ACTIVITIES IN THE COMMUNITY OF MADRID (SPAIN)}

\author{
Martínez-de-Quel-Pérez, Ó.1; Sánchez-Moreno, A.2; Zamorano-Feijóo, C.3; \\ Ayán-Pérez, C. ${ }^{4}$
}

\footnotetext{
1 Doctor en Ciencias de la Actividad Física y el Deporte; Universidad Complutense de Madrid; Facultad de Educación; Departamento de Didáctica de las Lenguas, Artes y Educación Física (España) odequel@ucm.es

2 Graduado en Ciencias de la Actividad Física y el Deporte y en Magisterio de Educación Primaria; Colegio Miramadrid; Departamentos de Extraescolares (España) asmca1990@gmail.com

${ }^{3}$ Graduado en Ciencias de la Actividad Física y el Deporte; Colegio Bilingüe Cantín y Gamboa; Departamento de Educación Física (España) carloszamoranofeijoo@gmail.com

4 Doctor en Ciencias de la Actividad Física y el Deporte; Universidad de Vigo; Facultad de Ciencias de la Educación y el Deporte; Departamento de Didácticas Especiales; Well-move Research Group (España) cayan@uvigo.es
}
Clasificación UNESCO / UNESCO code: 3202 Epidemiología / Epidemiology Clasificación Consejo de Europa / Council of Europe classification: 17. Otras: Epidemiología / Epidemiology

Recibido 1 de marzo de 2017 Received March 1, 2017

Aceptado 3 de mayo de 2018 Accepted May 3, 2018

\section{RESUMEN}

Este estudio tuvo como objetivo analizar la epidemiología lesional deportiva de las actividades desarrolladas bajo responsabilidad docente (Educación Física, recreo y actividades extraescolares) en centros de Educación Secundaria Obligatoria (ESO). Participaron 1.050 estudiantes (edad media 13,9 $\pm 1,3$ años) que cumplimentaron un cuestionario sobre lesiones deportivas. De las 848 lesiones registradas en total, 319 (172 en chicos y 147 en chicas) ocurrieron en actividades desarrolladas bajo responsabilidad docente $(0,30$ 
lesiones/estudiante). Las actividades extraescolares fueron el ámbito con mayor promedio de lesiones $(0,12$ por estudiante). Se observó una mayor incidencia lesional a los 13 años y una menor probabilidad de que las lesiones ocurriesen en los chicos $(O R=0,64(0,49-0,85) ; p<0,05)$. Los esguinces y las contusiones fueron las lesiones más frecuentes, especialmente en tobillo y rodilla. Un 27,3\% de las lesiones impidieron al alumnado participar activamente en las sesiones de Educación Física durante más de tres días.

PALABRAS CLAVE: lesiones, educación, deporte, profesor, actividades extraescolares.

\section{ABSTRACT}

This study aimed to analyse the epidemiology of sport injuries that occurred during activities performed under teacher supervision (Physical Education, break and extracurricular activities), at high school. A questionnaire regarding sport injuries were administered to 1050 students (mean age $13.9 \pm 1.3$ years). Out of the 848 sport injuries registered, 319 (172 in boys and 147 in girls) took place during activities performed under teacher supervision, $(0.30$ injuries/student). Extracurricular activities were the scenario in which the highest injury rate $(0.12)$ was observed. There was a higher incidence of injury at age 13 and a lower likelihood of injuries occurring in boys $(O R=0.64(0.49-0.85) ; p$ $<0.05)$. Sprains and contusions were the type of injury most frequently observed, especially in areas such as ankles and knees. A total of $27.3 \%$ of the injuries reported prevented the students from actively participating in the Physical Education sessions for more than three days.

KEYWORDS: injury, education, sport, teacher, extracurricular activities.

\section{INTRODUCCIÓN}

En la actualidad se ha constatado un incremento en la prevalencia del sedentarismo, especialmente en niños y adolescentes. Por lo tanto, se hace necesario establecer estrategias de promoción de la práctica de actividad física (AF) en edades tempranas (Arriscado, Muros, Zabala y Dalmau, 2014). A este respecto se ha observado que en este colectivo, el momento del día más propicio para la realización de AF tiene lugar en el horario escolar (De Baere, Lefevre, De Martelaer, Philippaerts y Seghers, 2015) y parece aconsejable desarrollar dichas estrategias en el propio centro educativo (Black, Menzel y Bungum, 2015). De hecho, distintas investigaciones han observado un aumento de la práctica de AF tras la realización de intervenciones promovidas por el centro educativo. Dichas intervenciones consistieron principalmente en aumentar el tiempo destinado a la Educación Física (EF) (Van Sluijs, McMinn y Griffin, 2007), facilitar la práctica de AF durante el recreo (Parrish, Okely, Stanley y Ridgers, 2013) y fomentar la participación en actividades extraescolares (Hatfield y Chomitz, 2015).

Sin embargo, es necesario tener en cuenta que el incremento de la práctica de la $\mathrm{AF}$ en niños y adolescentes conlleva también un aumento del 
riesgo de lesión (Nauta, Martin-Diener, Martin, Van Mechelen y Verhagen, 2015). De este modo, en estos tres ámbitos en los que la promoción de la AF se desarrolla bajo la responsabilidad del docente, existe cierta incidencia lesional que debe ser tenida en cuenta (Christiansen, Andersen, Ersbøll, Jespersen, Franz y Wedderkopp, 2014; Menckel y Laflamme, 2000; Prange y Kühr, 1990). Como consecuencia, se ha señalado la importancia que tiene la realización de intervenciones preventivas al respecto desde el propio centro educativo (Collard, Chinapaw y Van Mechelen, 2009). Este tipo de estrategias deben tener como punto de partida la identificación del riesgo de lesión deportiva que existe en los centros, así como el tipo y consecuencias que el sufrir este tipo de lesiones suponen en la vida académica del alumnado. Esta información puede ser de gran interés para los profesionales que trabajan en centros educativos, quienes juegan un importante papel a la hora de prevenir lesiones en la población escolar (Habelt, Hasler, Steinbrück y Majewski, 2011).

A pesar de su importancia, la evidencia científica sobre este tema no es abundante a nivel nacional (Onís, Varona, Gil, Felici y Embid, 2015), por lo que parece de interés el desarrollar más investigaciones sobre esta temática. Bajo estas circunstancias, el objetivo de este estudio fue aportar información sobre la prevalencia y tipo de lesión sufrida por el alumnado de Educación Secundaria Obligatoria de la Comunidad Autónoma de Madrid como consecuencia de la realización de AF durante las sesiones de EF, el recreo y las actividades extraescolares organizadas por el centro educativo.

\section{MÉTODO}

Investigación retrospectiva de carácter transversal.

\section{MUESTRA}

Para el diseño del presente estudio, se tuvo en cuenta que la probabilidad de que ocurran lesiones en los centros educativos está influenciada por su localización (urbano vs rural), tamaño del centro (número de clases) y características de las instalaciones deportivas (precarias vs adecuadas) (Sosnowska y Kostka, 2003; Grimaud, Piette, Clappier, Deguen y Pommier, 2007). En consecuencia, se seleccionaron cuatro centros educativos de la Comunidad Autónoma de Madrid de características diversas. Dos de ellos se encontraban en Madrid capital y los otros dos en municipios más pequeños dentro de esta región. El número de alumnos matriculados fue inferior a 600 en dos centros y superior a 1.200 en los otros dos. En cuanto a las instalaciones deportivas, dos centros contaban con pista exterior, gimnasio y pabellón, un centro tenía además piscina y pista polideportiva y otro centro tenía un pequeño gimnasio y una pista exterior de dimensiones reducidas.

Todo el alumnado que se encontraba cursando Educación Secundaria Obligatoria en estos centros durante el curso académico 2013/14 fue invitado a participar en este estudio. Aquellos estudiantes que presentaron algún tipo de problema de salud que les impidiese realizar EF durante el mencionado curso académico fueron excluidos del mismo. 


\section{INSTRUMENTOS}

Se utilizó un cuestionario que recogía información retrospectiva sobre la prevalencia y características de las lesiones deportivas ocurridas durante el curso escolar. Para la elaboración del mismo, se partió de una versión originalmente diseñada por Vaquero y González (2000) y posteriormente modificada por Gutiérrez-Castañón (2008 y 2014; Gutiérrez-Castañón, Martínezde-Haro, Ramos-Álvarez y Cid-Yagüe, 2018). Dicho cuestionario incluía preguntas sobre las características de las lesiones deportivas sufridas por el alumnado durante ese curso académico (tipo, zona corporal afectada, lesión recidivante), sobre el ámbito en el que ocurrió la lesión (sesión de EF, recreo, actividad extraescolar y otras), el tratamiento aplicado, el número de sesiones de EF en las que no se pudo participar de manera activa como consecuencia de la lesión (1, 2, 3 o más). Posteriormente se añadieron preguntas sobre el tipo de tarea a realizar durante el periodo de inactividad en las sesiones de EF y se modificaron las opciones a contestar en relación al tratamiento recibido, haciendo referencia a la persona a la que se acudió para tratar la lesión (Anexo I). Previamente a su administración, se realizó un estudio piloto en el que un grupo de 45 estudiantes de secundaria rellenaron el cuestionario en dos ocasiones con una semana entre ambas. Se comprobó que los estudiantes comprendían correctamente las preguntas y que en ambas ocasiones respondían de la misma manera.

\section{PROCEDIMIENTO}

El cuestionario fue administrado de manera grupal en el mes de mayo del curso académico 2013/14 durante las sesiones finales de EF, por dos Graduados en Ciencias de la AF y el Deporte. Previamente a su contestación, se detalló al alumnado el objetivo del estudio y se le explicó que el concepto de lesión deportiva hacía referencia a todo accidente o disfunción física acaecido durante la práctica deportiva, o como consecuencia directa de ella (Pascual, Pérez y Calvo, 2008). Para la realización del estudio, se solicitó por escrito el consentimiento informado a todos los participantes, a sus respectivos padresmadres-tutores y a los directores de los centros educativos. El estudio se realizó en cumplimiento de las normas de la Declaración de Helsinki (General Assembly of the World Medical Associaton, 2014) y siguiendo las directrices de la Comunidad Europea para la Buena Práctica Clínica (111/3976/88 de julio de 1990), así como el marco legal español para la investigación clínica en los seres humanos (Real Decreto 561/1993 sobre ensayos clínicos).

\section{ANÁLISIS ESTADÍSTICO}

Para realizar el análisis estadístico se utilizó el paquete SPSS 21. Inicialmente, tras el registro de datos y la eliminación de aquellos cuestionarios con errores en su cumplimentación, se contabilizaron el total de lesiones deportivas producidas en los diferentes ámbitos (EF, recreo, actividades extraescolares, fuera del centro escolar) en función del sexo y la edad y se hallaron promedios de lesiones por el número de estudiantes de cada grupo. La 
prueba ANOVA sirvió para conocer las diferencias entre grupos de edad, siendo utilizado el post-hoc de Games-Howell para conocer las diferencias ente los grupos dos a dos. El cálculo de Odds Ratio (OR) o razón de momios proporcionó el riesgo de lesionarse en función del sexo y el riesgo de que una lesión se produjera en uno u otro ámbito para cada sexo. El estadístico Chi-cuadrado se utilizó para determinar la existencia o no de diferencias significativas entre la frecuencia de lesiones por sexo, edad y ámbito. La descripción de las lesiones (tipo de lesión, localización anatómica), así como la persona a la que se acudió para su tratamiento, sus consecuencias en la pérdida de clases prácticas en EF y el trabajo realizado durante ese tiempo se realizó calculando el porcentaje de lesiones de cada tipo, utilizando el estadístico Chi-cuadrado para conocer si existían diferencias significativas entre la frecuencia de aparición de las mismas. Para todo el análisis, se estableció un nivel de significación $p<0,05$.

\section{RESULTADOS}

Se administró el cuestionario a un total de 1.075 alumnos, desechándose para un posterior análisis $25(2,3 \%)$ de ellos debido a errores en su cumplimentación, por lo que la muestra final estuvo conformada por 1.050 estudiantes (edad media 13,93 (SD = 1,3); mujeres 48\%). La distribución de los participantes por edad y sexo fue del siguiente modo: 12 años: 84 chicos y 83 chicas; 13 años: 138 chicos y 136 chicas; 14 años: 124 chicos y 116 chicas; 15 años: 111 chicos y 96 chicas; 16 años: 89 chicos y 73 chicas. En total se registraron 848 lesiones deportivas (515 en chicos y 333 en chicas), lo que supuso una media de 0,81 lesiones/estudiante. La distribución por ámbitos y sexo se observa en la tabla 1 . Un $37,6 \%$ de lesiones se registraron en actividades desarrolladas bajo responsabilidad docente (13,9\% en EF, 9,2\% en el recreo y $14,5 \%$ en Actividades Extraescolares) y un $62,4 \%$ en otro tipo de actividades realizadas fuera del ámbito educativo.

En este análisis global se observó la existencia de diferencias significativas entre los grupos de edad mediante la prueba de ANOVA ( $F=3,109$, $\mathrm{p}<0,05)$. El análisis post-hoc de Games-Howell confirmó que el número de lesiones en el grupo de 16 años era mayor que en los grupos de 12 y $13(p<0,05)$. En relación al sexo, el cálculo de OR mostró que el riesgo de lesionarse fue mayor en chicos que en chicas (OR 2,39 (1,86-3,08); $p<0,05)$, habiéndose lesionado durante ese curso académico el $68,7 \%$ de los chicos y el $47,8 \%$ de las chicas.

Tabla 1. Número de lesiones deportivas ocurridas en cada ámbito y distribuidas por sexo

\begin{tabular}{|l|c|c|c|}
\hline \multirow{2}{*}{$\begin{array}{l}\text { Ámbito donde tuvo lugar la } \\
\text { lesión }\end{array}$} & \multicolumn{2}{|c|}{ Sexo } & \multirow{2}{*}{ Total } \\
\cline { 2 - 3 } & Masculino & Femenino & \\
\hline Educación Física & 59 & 59 & 118 \\
\hline Recreo & 51 & 27 & 78 \\
\hline Extraescolar & 62 & 61 & 123 \\
\hline Fuera del ámbito educativo & 343 & 186 & 529 \\
\hline Total & 515 & 333 & 848 \\
\hline
\end{tabular}


El promedio de lesiones durante el curso académico se redujo a 0,30 lesiones/estudiante cuando solo se tuvieron en cuenta las 319 lesiones (172 en chicos y 147 en chicas) acontecidas en actividades desarrolladas bajo responsabilidad docente. En la tabla 2 se muestra el promedio de lesiones deportivas sufridas durante el curso escolar en EF, recreo y actividades extraescolares teniendo en cuenta la edad. Las actividades extraescolares fueron las prácticas en las que se observaron un mayor promedio de lesiones deportivas (0,12 por estudiante). Tomando como referencia estos tres ámbitos en su conjunto, el mayor índice de lesiones se registró a los 13 años y el menor a los 16 y a los 12 años. No se observó una evolución ni creciente ni decreciente del promedio de lesiones por estudiante en función de la edad.

Al analizar la influencia de la edad en cada uno de los tres ámbitos, el estadístico Chi-cuadrado $\left(x^{2}=19,75 ; p>0,05\right)$ mostró que algunas casillas de la tabla de contingencia estaban sobrerrepresentadas. Con el análisis de los residuos tipificados corregidos se comprobó que a los 12 años la frecuencia de lesiones en el recreo fue mayor y en actividades extraescolares fue menor de lo esperado. Por su parte, a los 15 años la frecuencia de lesiones en actividades extraescolares fue mayor de lo esperado si la distribución hubiese sido uniforme. En relación a la influencia del sexo, se observó que la probabilidad de que una lesión se produjese en las actividades realizadas bajo supervisión docente fue menor en chicos $(\mathrm{OR}=0,64(0,49-0,85) ; p<0,05)$. En el total de estos tres ámbitos (EF, recreo y actividades extraescolares), se comprobó que el $33,2 \%$ de las lesiones de los chicos y el $43,4 \%$ de las lesiones de las chicas tuvieron lugar en el ámbito educativo.

Analizando los tres ámbitos por separado, se encontró que el riesgo de que una lesión deportiva se presentase en EF y en actividades extraescolares fue mayor en las chicas (OR $0,78(0,49-1,23) \mathrm{p}<0,05$ y OR $0,79(0,50-1,25)$ $\mathrm{p}<0,05$ respectivamente), y mayor en los chicos en el caso del recreo (OR 1,87 $(1,10-3,18) p<0,05)$. La prueba Chi-cuadrado $\left(x^{2}=5,47 ; p>0,05\right)$ mostró que no existieron diferencias significativas en función del sexo en las frecuencias de los tres ámbitos analizados por separado.

Tabla 2. Promedio de lesiones deportivas en función de la edad y ámbito de práctica deportiva desarrollada bajo responsabilidad docente

\begin{tabular}{|l|c|c|c|c|c|}
\hline & \multicolumn{2}{|c|}{ Sexo } & \multicolumn{2}{c|}{ Ámbito donde tuvo lugar la lesión } \\
\hline $\begin{array}{l}\text { Edad del } \\
\text { alumno/a }\end{array}$ & $\begin{array}{c}\text { Masculin } \\
0\end{array}$ & $\begin{array}{c}\text { Femenin } \\
\text { o }\end{array}$ & $\begin{array}{c}\text { Educación } \\
\text { Física }\end{array}$ & $\begin{array}{c}\text { Recre } \\
0\end{array}$ & $\begin{array}{c}\text { Extraescola } \\
r\end{array}$ \\
\hline 12 años & 0,27 & 0,20 & 0,09 & 0,11 & 0,04 \\
\hline 13 años & 0,41 & 0,38 & 0,14 & 0,11 & 0,15 \\
\hline 14 años & 0,27 & 0,34 & 0,13 & 0,05 & 0,12 \\
\hline 15 años & 0,31 & 0,31 & 0,11 & 0,05 & 0,15 \\
\hline 16 años & 0,29 & 0,12 & 0,07 & 0,05 & 0,09 \\
\hline Total & 0,32 & 0,27 & 0,11 & 0,07 & 0,12 \\
\hline
\end{tabular}

El tipo de lesión deportiva registrada y las zonas anatómicas lesionadas con más frecuencia se muestran en las tablas 3 y 4 . Los esguinces y las 
contusiones fueron las lesiones más frecuentes tanto en general como por ámbitos, suponiendo el $34,8 \%$ y el $24,8 \%$ del total de las lesiones deportivas acontecidas durante la práctica de actividades realizadas bajo la responsabilidad docente. En lo referente a localización anatómica de las lesiones deportivas registradas en total y por ámbitos, el tobillo y la rodilla fueron las zonas corporales más frecuentemente afectadas con un $27 \%$ y un $17,9 \%$ respectivamente.

Tabla 3. Tipos de lesiones deportivas registradas durante la práctica de EF, Recreo y Actividades Extraescolares

\begin{tabular}{|c|c|c|c|c|c|}
\hline \multirow[b]{2}{*}{ Tipo de lesión } & \multicolumn{4}{|c|}{ Ámbito donde tuvo lugar la lesión } & \multirow{2}{*}{\begin{tabular}{|c|}
$\%$ del \\
total de \\
lesiones
\end{tabular}} \\
\hline & $\begin{array}{l}\text { Educación } \\
\text { Física }\end{array}$ & Recreo & Extraescolar & Total & \\
\hline Esguince & 45 & 28 & 38 & 111 & $34,80 \%$ \\
\hline Contusión & 32 & 20 & 27 & 79 & $24,76 \%$ \\
\hline $\begin{array}{l}\text { Rotura } \\
\text { muscular }\end{array}$ & 12 & 2 & 14 & 28 & $8,78 \%$ \\
\hline Fractura & 5 & 9 & 13 & 27 & $8,46 \%$ \\
\hline Quemadura & 6 & 7 & 10 & 23 & $7,21 \%$ \\
\hline Tendinitis & 4 & 3 & 8 & 15 & $4,70 \%$ \\
\hline Luxación & 2 & 0 & 7 & 9 & $2,82 \%$ \\
\hline Otras & 12 & 9 & 6 & 27 & $8,46 \%$ \\
\hline Total & 118 & 78 & 123 & 319 & $100,00 \%$ \\
\hline
\end{tabular}

Tabla 4. Localización anatómica de las lesiones deportivas registradas durante la práctica de EF, Recreo y Actividades Extraescolares

\begin{tabular}{|c|c|c|c|c|c|}
\hline \multirow{2}{*}{$\begin{array}{l}\text { Localización } \\
\text { anatómica de la } \\
\text { lesión }\end{array}$} & \multicolumn{4}{|c|}{ Ámbito donde tuvo lugar la lesión } & \multirow{2}{*}{$\begin{array}{c}\% \text { del total } \\
\text { de } \\
\text { lesiones }\end{array}$} \\
\hline & $\begin{array}{l}\text { Educación } \\
\text { Física }\end{array}$ & Recreo & Extraescolar & Total & \\
\hline Tobillo & 33 & 26 & 27 & 86 & $26,96 \%$ \\
\hline Rodilla & 17 & 17 & 23 & 57 & $17,87 \%$ \\
\hline Muñeca & 13 & 7 & 11 & 31 & $9,72 \%$ \\
\hline Espalda & 16 & 2 & 9 & 27 & $8,46 \%$ \\
\hline Mano & 4 & 6 & 14 & 24 & $7,52 \%$ \\
\hline $\mathrm{Pie}$ & 4 & 6 & 9 & 19 & $5,96 \%$ \\
\hline Cabeza & 4 & 5 & 3 & 12 & $3,76 \%$ \\
\hline Cuello & 6 & 0 & 3 & 9 & $2,82 \%$ \\
\hline Hombro & 3 & 1 & 2 & 6 & $1,88 \%$ \\
\hline Cadera & 2 & 1 & 0 & 3 & $0,94 \%$ \\
\hline Otras & 16 & 7 & 22 & 45 & $14,11 \%$ \\
\hline Total & 118 & 78 & 123 & 319 & $100,00 \%$ \\
\hline
\end{tabular}

Tras haber sufrido la lesión deportiva, el profesional a quien se dirigió el alumnado con mayor frecuencia fue el médico $(51,1 \%)$. En el otro extremo, las personas menos solicitadas para el tratamiento fueron el profesor $(1,88 \%)$ y el entrenador $(3,45 \%)$. Destaca que un importante número de lesiones fueron 
tratadas sin acudir a ninguna persona (18,5\%), acudiendo a un familiar $(10,34 \%)$ o por su cuenta $(10,34 \%)$.

Tabla 5. Persona a la que acudió para el tratamiento en función del lugar o escenario donde tuvo lugar la lesión

\begin{tabular}{|c|c|c|c|c|c|}
\hline \multirow{2}{*}{$\begin{array}{l}\text { Persona a la que acudió } \\
\text { para el tratamiento de la } \\
\text { lesión }\end{array}$} & \multicolumn{4}{|c|}{ Lugar o escenario donde tuvo lugar la lesión } & \multirow{2}{*}{$\begin{array}{l}\text { \% del } \\
\text { total de } \\
\text { lesiones }\end{array}$} \\
\hline & $\begin{array}{l}\text { Educación } \\
\text { Física }\end{array}$ & Recreo & Extraescolar & Total & \\
\hline Médico & 56 & 43 & 64 & 163 & $51,10 \%$ \\
\hline Ninguno & 27 & 14 & 18 & 59 & $18,50 \%$ \\
\hline Por su cuenta & 13 & 10 & 10 & 33 & $10,34 \%$ \\
\hline Familiar & 13 & 6 & 14 & 33 & $10,34 \%$ \\
\hline Entrenador & 0 & 0 & 11 & 11 & $3,45 \%$ \\
\hline Profesor & 4 & 2 & 0 & 6 & $1,88 \%$ \\
\hline Otro & 5 & 3 & 6 & 14 & $4,39 \%$ \\
\hline Total & 118 & 78 & 123 & 319 & $100,00 \%$ \\
\hline
\end{tabular}

Teniendo en cuenta el ámbito donde se produjo la lesión y la persona a la que el estudiante acudió para el tratamiento de la sesión se calculó el estadístico Chi-cuadrado $\left(X^{2}=26,20, p<0,05\right)$, que mostró que existía alguna casilla sobrerepresentada en la tabla de contingencia. El cálculo post-hoc de los residuos tipificados corregidos mostró que, de manera estadísticamente significativa, la persona lesionada acudió al entrenador cuando la lesión se produjo en el ámbito extraescolar pero no lo hizo cuando se produjo durante una clase de Educación Física.

En la figura 1 se muestran las consecuencias que la lesión deportiva tuvo en la participación en EF. Un $39,6 \%$ de las lesiones sufridas no limitaron la práctica de EF, mientras que el $27,3 \%$ de las mismas supusieron que el alumnado no pudiera participar de forma activa durante las mismas durante más de tres sesiones consecutivas.

Figura 1. Consecuencias de las lesiones deportivas en la participación en EF 


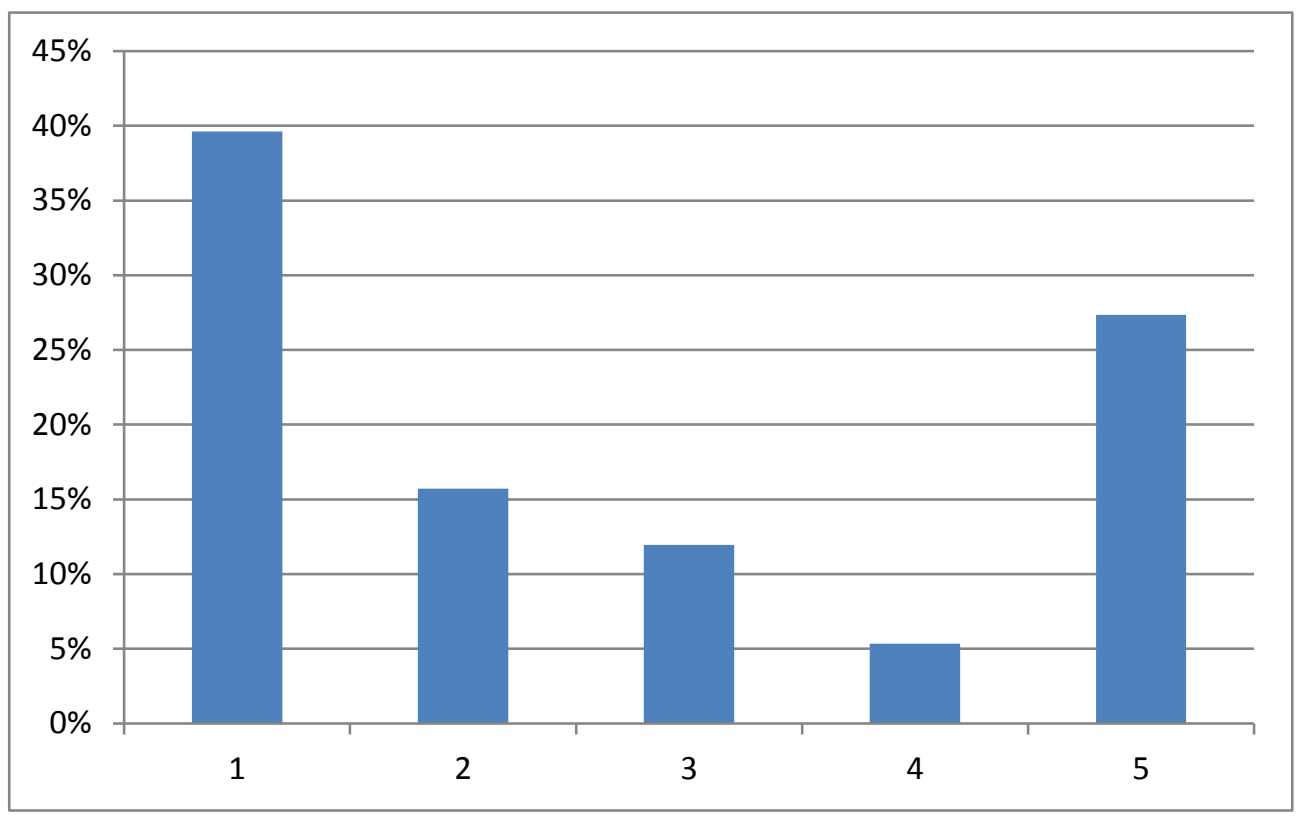

En relación a las actividades realizadas por el alumnado cuando la lesión deportiva le impidió participar de manera activa en las sesiones de EF (Figura 2 ), las tareas de observación (52,5\% de los casos) y el desarrollo de un diario de clase $(24,5 \%)$ fueron las más frecuentemente realizadas. En un $12 \%$ de los casos no se le asignó tarea alguna al alumnado.

Figura 2. Tareas asignadas al alumnado exento de la participación activa en EF por causa de lesión deportiva

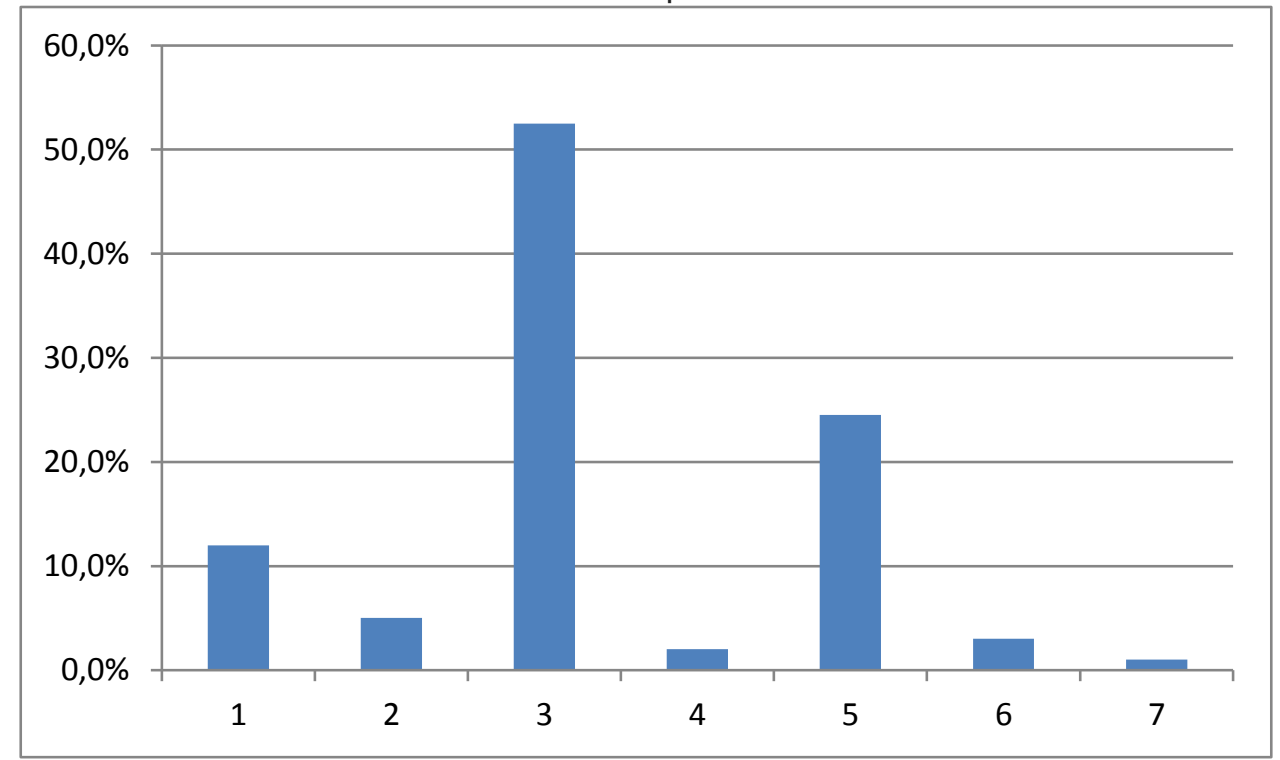

\section{DISCUSIÓN}

El propósito de este estudio fue aportar información sobre las características y tipos de lesiones que se producen en tres ámbitos fundamentales en los que la práctica de AF es promovida bajo la responsabilidad docente: EF, recreo y actividades extraescolares. Las investigaciones 
desarrolladas a este respecto en el contexto nacional son escasas y no están exentas de limitaciones. Así, Vaquero et al. (2000) informaron sobre el número de lesiones observadas en el centro educativo, pero la muestra de estudio fue pequeña $(n=123)$ y no se identificaron de manera específica las lesiones ocurridas durante los recreos. Más recientemente, Gautier y Martínez (2011) analizaron las lesiones ocurridas en varios centros educativos, pero no diferenciaron entre lesión deportiva y accidente, y tampoco distinguieron los ámbitos en los que la lesión tuvo lugar. Finalmente, Onís et al. (2016) describieron el número, tipo y gravedad de lesiones ocurridas durante el horario escolar, pero solo reportaron información sobre aquellas que habían sido tratadas en un servicio de emergencias.

Quizás el trabajo de investigación más completo realizado hasta la fecha sea la tesis doctoral de Gutiérrez-Castañón (2014), en la que se comparó el porcentaje de lesiones acaecido dentro y fuera de las sesiones de EF. Este autor también empleó un cuestionario de recuerdo, administrado a una muestra considerablemente menor a la del presente estudio (637 alumnos de $4^{\circ}$ de la ESO). Sin embargo, se debe reconocer que en dicha investigación se empleó una metodología más robusta, puesto que se controlaron de manera objetiva las faltas de asistencia y se tuvieron en cuenta dos aspectos fundamentales. En primer lugar, el cuestionario se administró de manera trimestral, reduciendo así el posible sesgo de recuerdo. En segundo lugar, se obtuvo información sobre la cantidad y tipo de AF que realizaban los alumnos fuera de su horario escolar, variable que puede tener una incidencia directa sobre la posibilidad de sufrir una lesión en el centro educativo.

Por ello, aunque los resultados aquí presentados contribuyen a incrementar y completar la evidencia científica existente al respecto en nuestro país, se debe tener en cuenta que los mismos fueron obtenidos a través de un diseño metodológico no exento de debilidades. Este hecho limita la fortaleza, transferencia en interpretación de los datos obtenidos, que requieren ser complementados por los ya existentes y por futuras investigaciones.

Del análisis realizado se desprende que la práctica de AF en el centro escolar y en actividades extraescolares organizadas desde el mismo es relativamente segura, sobre todo si se compara con la AF realizada en otros ámbitos que no se encuentran bajo la responsabilidad docente. Estos datos concuerdan con lo observado por Gutiérrez-Castañón, Valbuena-Láiz, ÁlvarezBarrio, Cid-Yagüe, Martínez-de-Haro y Muñoa-Blas (2007), quien tras registrar el número de lesiones ocurridas en 926 alumnos de $1^{\circ}$ y $3^{\circ}$ de la ESO durante un mes, encontró que del total de 31 escolares que se habían lesionado, tan solo 8 sufrieron la lesión durante la práctica de EF. Este hecho pudiera ser debido a que tanto la $A F$ que se realiza en otros ámbitos, ya sea desorganizada y libre como la que se practica de manera reglada y competitiva, tienen un índice lesional elevado y superior al observado en la práctica deportiva de los centros educativos (Nauta et al., 2015).

Aunque en comparación con otros hábitats como la calle o el hogar, el centro educativo muestra una siniestralidad mucho menor (Navascues et al., 2005; Benítez, Soriano y León, 2010), en el presente estudio se ha constatado 
una frecuencia lesional que no debe pasar desapercibida, lo que está en línea con lo observado en otros estudios. Así por ejemplo, Sundblad, Saartok, Engström y Renström (2005) observaron un total de 306 lesiones deportivas reportadas por una muestra de 1.975 niños a lo largo de un trimestre, de las cuales un $25 \%(n=77)$ y un $16 \%(n=48)$ ocurrieron durante las sesiones de EF y el recreo. En esta línea, Prange y Kühr, (1990) reportaron que de un total de 887 accidentes escolares atendidos en un servicio de emergencia, un 53\% ocurrió durante sesiones dirigidas al aprendizaje deportivo y un $28 \%$ durante los recreos.

En la literatura existe evidencia científica que apunta a que la práctica de EF constituye un importante factor de riesgo lesional (Carmeli, Azencot, Wertheim y Coleman, 2003; Peltzer y Pengpid, 2015) que incluso puede representar hasta un cuarto del total de los accidentes escolares (Nelson, Alhajj, Yard, Comstock y McKenzie, 2009). Sin embargo, la incidencia lesional derivada de la práctica de EF encontrada en este estudio se puede considerar como reducida. Posibles motivos que contribuirán a esta baja incidencia podrían ser la frecuencia bisemanal de las sesiones, el que las clases se desarrollen bajo la responsabilidad de docentes con formación específica para impartir la materia y a la intensidad controlada bajo la que por lo general se suelen realizar las mismas en la ESO (Greier, Heinzle, Nepo, Ratschiller, Gafriller y Riechelmann, 2015; Christiansen et al., 2014).

En contraposición a lo observado en EF, sí se constató una elevada incidencia lesional en actividades extraescolares desarrolladas bajo la responsabilidad del centro. En esta línea, en uno de los escasos estudios nacionales que aportaron información al respecto y realizado con 401 alumnos de $4^{\circ}$ de la ESO seguidos durante un trimestre, se encontró que eran las actividades extraescolares las que presentaban un mayor número de lesiones, con una importante diferencia sobre las clases de Educación Física y el deporte federado (Gutiérrez-Castañón, 2008). A nivel internacional, Prange y Khur (1990) encontraron que el $3 \%$ de los accidentes escolares tenían lugar durante este tipo de actividades, mientras que Sun, Yu, Wong, Zhang, Fan y Guo (2006) encontraron un porcentaje bastante más elevado $(22,8 \%)$, siendo además el ámbito escolar en el que mayor número de lesiones se observaron, al igual que en el presente estudio. Este elevado número de lesiones podría estar motivado por dos razones. En primer lugar, es frecuente la organización por parte de los centros educativos de actividades extraescolares basadas en la realización de prácticas deportivas alternativas (p.ej. semana blanca, actividades en el medio natural) y que pudieran presentar un riesgo lesional alto. Por otro lado, también es posible que el alumnado se lesionase durante la práctica deportiva organizada por los centros docentes en horario extraescolar ( $p$. ej. iniciación deportiva, competiciones inter 0 intra-centros). El elevado porcentaje de lesiones encontrado en este estudio aconseja tener en cuenta las actividades extracurriculares supervisadas a la hora de desarrollar estrategias de prevención de accidentes deportivos, sugerencia previamente advertida por otros autores (Pressley, Barlow, Durkin, Jacko, Domínguez y Johnson, 2005).

Dos factores que están asociados a la epidemiología lesional son la edad y el sexo. A este respecto, algunos autores han informado de que la influencia de ambos no es significativa en la frecuencia de lesiones registradas en el ámbito 
docente (Sunblad et al., 2005; Vaquero y González, 2000). En el presente estudio se identificó un promedio de lesiones más elevado a los 13 años de edad para ambos sexos al analizar conjuntamente las lesiones ocurridas en EF, recreo y actividades extraescolares. Este dato coincide con lo reportado por Sosnowska y Kostka (2003), quienes registraron un pico de incidencia lesional en los centros educativos a esta misma edad. Además, cuando se ha estudiado conjuntamente el sexo y la edad, se ha encontrado una mayor frecuencia de aparición de lesiones deportivas a los 12 años en general, existiendo mayor número de lesiones entre los 13-14 años en chicas y entre los 15-16 en chicos (Abernethy y MacAuley, 2003).

Otro aspecto destacable de los resultados obtenidos en esta investigación, es el hecho de que el riesgo relativo de que las lesiones deportivas ocurriesen en actividades desarrolladas bajo responsabilidad docente fue menor en los chicos, lo que está en línea con lo reportado por otros autores. Así, Nauta et al. (2014), centrando su análisis en la práctica deportiva escolar, informaron de un índice más elevado de lesión en las chicas. De manera similar, Verhagen, Collard, Paw y Van Mechelen (2009), también observaron una mayor probabilidad de accidente deportivo en las niñas, al analizar el total de lesiones acontecidas durante un curso académico en escolares de 10-12 años de edad. Factores como la menor habilidad deportiva o el menor nivel de condición física que pudieran presentar las chicas pudieran ser los motivos que explicasen esta situación. Igualmente, es destacable el hecho de que el riesgo de que una lesión deportiva ocurriese durante la práctica de EF fue mayor en las chicas. Este hallazgo, concuerda con los de otros autores que han observado un mayor porcentaje de lesiones durante las sesiones en EF en el género femenino (Sunbland et al., 2005; Videmsek, Karpljuk, Mlinar, Meško y Štihec, 2010).

En relación al tipo de lesiones y localización de las mismas, los esguinces fueron las lesiones más frecuentes y las extremidades inferiores las más afectadas, al igual que se ha observado en investigaciones similares (Sunblad et al., 2005; Carmeli et al., 2003; Collard, Chinapaw y Van Mechelen, 2009). Este patrón, que se hizo igualmente patente durante las sesiones de EF, también coincide con la literatura previa publicado al respecto (Gutiérrez-Castañón, 2014; Nelson et al., 2009).

Generalmente existe poca información disponible sobre las consecuencias que las lesiones deportivas ocurridas en actividades desarrolladas bajo la responsabilidad docente tienen sobre la participación del alumnado lesionado en las sesiones de EF. A este respecto, Gutiérrez-Castañón (2014), observó que las lesiones producidas fuera de las clases de EF suponían un mayor periodo de inactividad, recalcando que los alumnos que se lesionaban durante la práctica de EF no solían ausentarse más de un día. Los resultados del presente estudio indicaron que ninguna lesión supuso la exención de la asignatura, pero sí que un porcentaje considerable de las mismas (27\%) tuvo como consecuencia que el alumnado no pudiese participar activamente y de manera consecutiva en tres o más sesiones de EF. Este dato, unido al hecho de que hasta en un $12 \%$ de los casos al alumnado no se le asignó tarea alguna durante las sesiones de EF, es de cierto interés para la práctica docente. Se ha comprobado que las consecuencias académicas de las lesiones deportivas 
sufridas en actividades desarrolladas bajo la responsabilidad docente tienen una magnitud considerable, ya que la lesión no solo limita la adquisición de contenidos por pate del alumnado de manera práctica, sino también la posibilidad de reforzar los conocimientos mediante tareas alternativas relacionadas con la temática impartida.

Finalmente, cabe recordar la importancia que tiene para el docente no solo identificar en qué actividades se lesionan más sus alumnos y las características de las mismas, sino también el saber actuar convenientemente al respecto (Barrios, Jones y Gallagher, 2007). Los resultados de este estudio apuntan a que el profesor no es la primera persona a la que se recurre en el caso de las lesiones ocurridas bajo la responsabilidad docente, lo que está en línea con lo reportado en otros estudios (Sunbland et al., 2005). Pese a que no es frecuente que el alumnado reciba tratamiento médico en el propio centro (Onís et al., 2015), estos hallazgos ahondan en la necesidad previamente observada de que el profesorado adquiera conocimientos básicos que le permitan actuar con solvencia en este tipo de situaciones (Abernethy, MacAuley, McNally y McCann, 2003).

A pesar de la originalidad de esta investigación y de la utilidad que sus hallazgos puedan tener a la hora de desarrollar estrategias de prevención, existen ciertas debilidades metodológicas que limitan la generalización y aplicabilidad de los mismos. Esto es debido principalmente a que se empleó un cuestionario para registrar el número de lesiones producidas, metodología frecuente empleada en estudios similares (Gutiérrez-Castañón, 2014). Sin embargo, el tiempo de recuerdo solicitado fue de un año, por lo que no se puede desechar la existencia de un sesgo marcado por la capacidad para recordar con efectividad toda la información solicitada sobre la misma al alumnado. Por otro, la muestra de este trabajo estuvo conformada exclusivamente por alumnado de la Comunidad de Madrid, por lo que los resultados encontrados pudieran no ser directamente extrapolables a centros educativos de otras Comunidades Autónomas. Tales centros pudieran tener otra organización de la práctica deportiva en función de factores como el horario escolar, la climatología y los tipos de actividades extraescolares organizadas que se practican. Finalmente, se debe reconocer que el haber contado con un mayor número de centros y por tanto de alumnos, dotaría de una mayor potencia estadística a los resultados obtenidos, siendo por lo tanto las conclusiones extraídas más robustas.

En conclusión, los resultados de este estudio indican que las lesiones deportivas ocurridas en actividades realizadas bajo responsabilidad docente suceden en menor medida en comparación con las observadas en otros ámbitos en los que el alumnado práctica AF. El riesgo de que este tipo de lesiones tengan lugar en el ámbito educativo es menor en los chicos y la frecuencia lesional es mayor a los 13 años. Las lesiones deportivas más frecuentes son los esguinces y las contusiones, especialmente en el tobillo y la rodilla, observándose que su gravedad en algunos casos puede limitar la participación activa del alumnado en tres o más sesiones de EF. Las estrategias de prevención deben prestar especial atención al desarrollo de actividades extraescolares, pues presentan un mayor porcentaje lesional en comparación con la EF y el recreo respectivamente. 


\section{REFERENCIAS BIBLIOGRÁFICAS}

Abernethy, L. \& MacAuley, D. (2003). Impact of school sports injury. British Journal of Sports Medicine, 37(4), 354-355. https://doi.org/10.1136/bjsm.37.4.354

Abernethy, L., MacAuley, D., McNally, O. \& McCann, S. (2003). Immediate care of school sport injury. Injury prevention, 9(3), 270-273. https://doi.org/10.1136/ip.9.3.270

Arriscado, D., Muros, J. J. Zabala, M. \& Dalmau, J. M. (2014). Hábitos de práctica física en escolares: factores influyentes y relaciones con la condición física. Nutrición Hospitalaria, 31(3), 1232-1239.

Barrios, L. C., Jones, S. E. \& Gallagher, S. S. (2007). Legal liability: The consequences of school injury. Journal of school health, 77(5), 273-279. https://doi.org/10.1111/j.1746-1561.2007.00203.x

Benítez, B. R., Soriano, M. \& León, A. C. (2010). Prevención de la accidentalidad infantil en Andalucía: aprender a crecer con seguridad. Anales de Pediatría, 73(5), 249-256. https://doi.org/10.1016/j.anpedi.2010.03.009

Black, I. E., Menzel, N. N. \& Bungum, T. J. (2015). The Relationship Among Playground Areas and Physical Activity Levels in Children. Journal of Pediatric Health Care, 29(2), 156-168. https://doi.org/10.1016/j.pedhc.2014.10.001

Carmeli, E., Azencot, S., Wertheim, M. \& Coleman, R. (2003). Sports injuries in students aged 12-18 during physical education classes in Israel. Biology of Sport, 20(4), 271-280.

Christiansen, C., Andersen, L. B., Ersbøll, A. K., Jespersen, E., Franz, C. \& Wedderkopp, N. (2014). Injuries in children with extra physical education in primary schools. Medicine and science in sports and exercise, 46(4), 745-752. https://doi.org/10.1249/MSS.0000000000000152

Collard, D. C., Chinapaw, M. J. \& Van Mechelen, W. (2009). Design of the iPlay Study. Sports medicine, 39(11), 889-901. https://doi.org/10.2165/11317880-000000000-00000

De Baere, S., Lefevre, J., De Martelaer, K., Philippaerts, R. \& Seghers, J. (2015). Temporal patterns of physical activity and sedentary behavior in 10-14 year-old children on weekdays. BMC public health, 15(1), 1. https://doi.org/10.1186/s12889-015-2093-7

Greier, K., Heinzle, A., Nepo, S., Ratschiller, J., Gafriller, R. \& Riechelmann, H. (2015). [Safety in Physical Education-A Teacher's Perspective]. Sportverletzung Sportschaden: Organ der Gesellschaft fur OrthopadischTraumatologische Sportmedizin, 29(1), 40-45.

Grimaud, O., Piette, C., Clappier, P., Deguen, S. \& Pommier, J. (2007). Accidents en éducation Archives de pédiatrie, 14(4), 391-393.

Gutiérrez-Castañón, E. (2014). Epidemiología de las lesiones deportivas en clase de Educación Física (Tesis doctoral). Universidad Autónoma de Madrid.

Gutiérrez-Castañón E. (2008). Lesiones y falta de actividad en Educación Física. Madrid: CV Ciencias del Deporte.

Gutiérrez-Castañón, E.; Martínez-de-Haro, V.; Ramos-Álvarez, J.J. y Cid-Yagüe, L. (2018) Injuries in Physical Education of High School. A Problem? 
Revista Internacional de Medicina y Ciencias de la Actividad Física y el Deporte, 18 (72), 709-722 http://doi.org/10.15366/rimcafd2018.72.007

Gutiérrez-Castañón, E., Valbuena-Láiz, C., Álvarez-Barrio MJ., Cid-Yagüe L., Martínez-de-Haro, V. \& Muñoa-Blas J. (2007). Causas de absentismo en Educación Física en ESO, Selección, 16(2), 84-90.

Habelt, S., Hasler, SC., Steinbrück, K. \& Majewski, M. (2011). Sport injuries in adolescents. Orthop Rev, 3(18), 82-86. https://doi.org/10.4081/or.2011.e18

Hatfield, D. P. \& Chomitz, V. R. (2015). Increasing Children's Physical Activity During the School Day. Current obesity reports, 4(2), 147-156. https://doi.org/10.1007/s13679-015-0159-6

Menckel, E. \& Laflamme, L. (2000). Injuries to boys and girls in Swedish schools: different activities, different results?. Scand J Public Health, 28(2), 132-6. https://doi.org/10.1177/140349480002800209

Nauta, J., Martin-Diener, E., Martin, B. W., Van Mechelen, W. \& Verhagen, E. (2015). Injury risk during different physical activity behaviours in children: A systematic review with bias assessment. Sports medicine, 45(3), 327336. https://doi.org/10.1007/s40279-014-0289-0

Navascués, J. A., Matute, J., Soleto, J., García, C. M., Hernández, E., SánchezParís, O., ... \&Vázquez, J. (2005). Paediatric trauma in Spain: a report from the HUGM Trauma Registry. EurJ Pediatr Surg, 15(1), 30-37. https://doi.org/10.1055/s-2004-821213

Nelson, N. G., Alhajj, M., Yard, E., Comstock, D. \& McKenzie, L. B. (2009). Physical education class injuries treated in emergency departments in the US in 1997-2007. Pediatrics, 124(3), 918-925. https://doi.org/10.1542/peds.2008-3843

Onís González, E., Varona Pérez, I., Gil Pérez, M., Felici, C. \& Embid Pardo, P. (2015). Lesiones no intencionadas en el centro escolar: ¿ de qué estamos hablando?. Pediatría Atención Primaria, 17(68), 333-339. https://doi.org/10.4321/S1139-76322015000500008

Parrish, A. M., Okely, A. D., Stanley, R. M. \& Ridgers, N. D. (2013). The effect of school recess interventions on physical activity. Sports Medicine, 43(4), 287-299. https://doi.org/10.1007/s40279-013-0024-2

Pascual, C. M., Pérez, V. R. \& Calvo, J. S. (2008). Epidemiología de las lesiones deportivas. Fisioterapia, 30(1), 40-48. https://doi.org/10.1016/S02115638(08)72954-7

Peltzer, K. \& Pengpid, S. (2015). Unintentional injuries and psychosocial correlates among in-school adolescents in Malaysia. International journal of environmental research and public health, 12(11), 14936-14947. https://doi.org/10.3390/ijerph121114936

Prange, C. H. \& Kühr, J. (1990). Accident and injuries situations in accidents at school and on the way to and from school. Der Unfallchirurg, 93(8), 346352.

Pressley, J. C., Barlow, B., Durkin, M., Jacko, M. S. A., Dominguez, M. D. R. \& Johnson, M. L. (2005). A national program for injury prevention in children and adolescents: the injury free coalition for kids. Journal of Urban Health, 82(3), 389-402. https://doi.org/10.1093/jurban/jti078 
Sosnowska, S. \&Kostka, T. (2003). Epidemiology of school accidents during a six school-year period in one region in Poland. Eur J Epidemiol, 18(10), 977982. https://doi.org/10.1023/A:1025802203726

Sun, Y. H., Yu, I. T. S., Wong, T. W., Zhang, Y., Fan, Y. P. \& Guo, S. Q. (2006). Unintentional injuries at school in China patterns and risk factors. Accident Analysis \& Prevention, 38(1), 208-214. https://doi.org/10.1016/j.aap.2005.09.010

Sundblad, G., Saartok, T., Engström, L. M. \& Renström, P. (2005). Injuries during physical activity in school children. Scandinavian journal of medicine \& science in sports, 15(5), 313-323. https://doi.org/10.1111/j.16000838.2004.00419.x

Van Sluijs, E. M., McMinn, A. M. \& Griffin, S. J. (2007). Effectiveness of interventions to promote physical activity in children and adolescents: systematic review of controlled trials. Bmj, 335(7622), 703. https://doi.org/10.1136/bmj.39320.843947.BE

Gautier Vargas, M.\& Martínez González, V. (2011). Accidentes y lesiones más frecuentes en edad escolar. Rev Rol Enferm, 34(1), 26-31.

Vaquero, M. \& González, J. (2000). Utilidad del registro de lesiones deportivas en el marco escolar. Apunts. Medicina de l'Esport, 35(133), 13-18. https://doi.org/10.1016/S1886-6581(00)75957-3

Verhagen, E., Collard, D., Paw, M. C. \& Van Mechelen, W. (2009). A prospective cohort study on physical activity and sports-related injuries in 10-12 yearold children. $\mathrm{Br} J$ Sports Med, 43(13), 1031-1035. https://doi.org/10.1136/bjsm.2008.055483

Videmšek, M., Karpljuk, D., Mlinar, S., Meško, M. \& Štihec, J. (2010). Injuries to primary school pupils and secondary school students during physical education classes and in their leisure time. Collegium antropologicum, 34(3), 973-980.

Número de citas totales / Total references: $36(100 \%)$

Número de citas propias de la revista / Journal's own references: 1 (2,7\%)

Rev.int.med.cienc.act.fís.deporte - vol. 19 - número 73 - ISSN: 1577-0354 